\title{
Endoscopic Endonasal Surgery for Subdiaphragmatic Type Craniopharyngiomas
}

\author{
Hiroshi NISHIOKA, ${ }^{1,2}$ Yuichi NAGATA, ${ }^{1}$ Noriaki FUKUHARA, ${ }^{1}$ \\ Mitsuo YAMAGUCHI-OKADA, ${ }^{1}$ and Shozo YAMADA $^{1,2}$ \\ ${ }^{1}$ Department of Hypothalamic and Pituitary Surgery, Toranomon Hospital, Tokyo, Japan; \\ ${ }^{2}$ Okinaka Memorial Institute for Medical Research, Tokyo, Japan
}

\begin{abstract}
Subdiaphragmatic type craniopharyngiomas are tumors that originate within the sella. They are divided into two types; those localized within an enlarged sella (intrasellar type) and those accompanying a suprasellar extension (suprasellar extended type). The clinicopathological features and the recent outcomes of endoscopic endonasal surgery were retrospectively reviewed in 32 patients, with 11 surgeries for recurrence. These tumors showed a preponderance in young patients (19 patients were younger than 18-year-old) and suprasellar extended type (25 cases), were mostly composed of a large cyst $(96.9 \%)$ and were frequently adamantinomatous type $(68.8 \%)$. Combined transcranial-endoscopic endonasal surgery was applied in three patients with extremely large tumors and significant frontal extension. Total tumor resection and stalk preservation were achieved in 26 and 17 patients, respectively. No complications developed after surgery apart from pituitary dysfunction and visual deterioration. 5 of 6 patients with subtotal tumor resection and 6 of 7 patients with no improvement or deterioration of visual function were in the recurrent cases. Although this type is basically an extraarachnoidal tumor, the suprasellar portion of the tumor showed adherence to important tissues in some patients with recurrence. Pituitary function remained normal in only one third of patients with stalk preservation. To avoid pituitary dysfunction after surgery, sharp excision of firm adherence to the stalk should be considered in some patients.
\end{abstract}

Key words: craniopharyngiomas, endoscopic endonasal surgery, pituitary function, subdiaphragmatic type

\section{Introduction}

Treatment of craniopharyngioma, a deep-seated benign tumor, has been one of the biggest challenges confronting neurosurgeons since the era of Harvey Cushing. Thanks to the recent refinements in surgical instruments and techniques, many of these tumors can be safely and reliably resected by endoscopic endonasal surgery (EES) at present. ${ }^{1-4)}$ Overall survival is significantly improved, but endocrinological outcome remains unsatisfactory in many patients. The surgical approach mainly depends on tumor location. For those originating within the sella (subdiaphragmatic type), conventional transsphenoidal surgery (TSS) has been used as the firstline surgical approach before the development of EES. ${ }^{5-8)}$ In general, this type of tumor is considered to have a higher chance of radical resection and

Received February 2, 2018; Accepted April 5, 2018

Copyright $\odot 2018$ by The Japan Neurosurgical Society This work is licensed under a Creative Commons AttributionNonCommercial-NoDerivatives International License. stalk preservation after surgery with a low risk of complications. We have been using TSS and EES as primary surgical treatments for most patients with craniopharyngioma irrespective of age. ${ }^{9)}$ In the present retrospective study, the recent series of EES for subdiaphragmatic type craniopharyngioma was evaluated to elucidate surgical outcomes, particularly the endocrinological result.

\section{Methods}

\section{Patients}

The cases of 32 consecutive patients with subdiaphragmatic type craniopharyngioma surgically treated between January 2014 and April 2017 are reviewed herein. Subdiaphragmatic type was defined as those tumors that originated within the sella. It was divided into two types; the type with the tumor localized within an enlarged sella (intrasellar type; IS type, 7 cases) and the type with the tumor accompanying a suprasellar extension (suprasellar extended type; SSE type, 25 cases) (Table 1). They made up $30.2 \%$ of the 106 craniopharyngioma 
patients who underwent EES during this period at our institute. The 16 male and 16 female patients ranged in age from 11 months to 69 (mean 27.4) years. 19 patients $(59.4 \%)$ were younger than 18-year-old. Surgery was performed for the first time in 21 patients, whereas 11 patients (34.4\%) had previously undergone surgical treatment for the tumor, either TSS (6 patients) or transcranial (5 patients), at other hospitals (9 patients) or at our hospital (2 patients) (Table 2). 3 of 11 patients

Table 1 Differences between intrasellar type and suprasellar extended type tumors

\begin{tabular}{lcc}
\hline & $\begin{array}{c}\text { Intrasellar } \\
\text { type } \\
(n=7)\end{array}$ & $\begin{array}{c}\text { Suprasellar } \\
\text { extended type } \\
(n=25)\end{array}$ \\
\hline Age (<18 years old) & 3 & 16 \\
Gender (male/female) & $2 / 5$ & $14 / 11$ \\
Initial/Repeat surgery & $6 / 1$ & $15 / 10$ \\
Convent/Extend/Comb & $5 / 2 / 0$ & $6 / 16 / 3$ \\
Ad/Pap/Cil & $3 / 4 / 0$ & $19 / 3 / 3$ \\
Total/Subtotal resection & $7 / 0$ & $19 / 6$ \\
Stalk preserved & 3 & 14 \\
VD pre/postop. & $4 / 1$ & $23 / 6$ \\
Hypopit pre/postop. & $5 / 5$ & $20 / 22$ \\
DI pre/postop. & $2 / 4$ & $7 / 22$ \\
\hline Ad: ang
\end{tabular}

Ad: adamantinematous type, Cil: ciliated type, Comb: combined transcranial and EES, Convent: conventional EES, DI: diabetes insipidus, Extend: extended EES, Hypopit: hypopituitarism, Pap: papillary type, VD: visual disturbance.

Table 2 Differences between patients who underwent primary surgery and repeat surgery

\begin{tabular}{lcc}
\hline & $\begin{array}{c}\text { Patients with } \\
\text { primary surgery } \\
(n=21)\end{array}$ & $\begin{array}{c}\text { Patients with } \\
\text { repeat surgery } \\
(n=11)\end{array}$ \\
\hline Age (<18) & 13 & 6 \\
Gender (male/female) & $11 / 10$ & $5 / 6$ \\
IS/SSE & $6 / 15$ & $1 / 10$ \\
Convent/Extend/Comb & $10 / 8 / 3$ & $1 / 10 / 0$ \\
Ad/Pap/Cil & $14 / 5 / 2$ & $8 / 2 / 1$ \\
Total/Subtotal & $20 / 1$ & $6 / 5$ \\
resection & & \\
Stalk preserved & 12 & 5 \\
VD pre/postop. & $17 / 1$ & $10 / 6$ \\
Hypo pre/postop. & $15 / 17$ & $10 / 10$ \\
DI pre/postop. & $5 / 16$ & $4 / 10$ \\
\hline Ad: adamantingm
\end{tabular}

Ad: adamantinematous type, Cil: ciliated type, Convent: conventional EES, DI: diabetes insipidus, Extend: extended EES, Comb: combined transcranial and EES, Hypopit: hypopituitarism, IS: intrasellar type, Pap: papillary type, SSE: suprasellar extended type, VD: visual disturbance. had undergone adjuvant stereotactic radiosurgery previously.

\section{Surgical approaches and assessments}

Conventional EES was performed in 11 patients (34.4\%), an extended EES (transtuberculum sellae approach) in 18 patients (56.3\%), and a simultaneous combined transcranial-EES approach in 3 patients $(9.4 \%$, Fig. 1). In patients with a high-flow cerebrospinal fluid leak after tumor resection, the sella was reconstructed by a multilayer method using a septal flap combined with fascial graft dural suturing. ${ }^{10)}$

In every patient, postoperative Magnetic resonance imaging (MRI) was performed within 3 days and between 3 and 6 months after surgery. The degree of tumor resection was determined by estimation by both surgeons and radiologists as follows: total and subtotal (more than 90\%). After surgical intervention, every patient was followed with clinical symptoms, endocrinological examinations, and MRI every 6 months. The follow-up period ranged from 9 to 48 (30.2) months.

\section{Results}

\section{CT and MRI findings and histology}

Tumor size ranged from 16.5 to $63.3(31.2) \mathrm{mm}$. The cystic component was noted within the tumor in 31 cases (96.9\%), 18 (56.3\%) of which were almost an entirely cystic tumor. On bone-window Computed tomography (CT), calcification of the tumor was noted in 28 cases $(87.5 \%)$. In 25 cases with SSE type, the pituitary stalk was always dorsal of the tumor and the tumor showed significant frontal or subfrontal extensions in 13 cases. 5 of them had a large cystic tumor exceeding $40 \mathrm{~mm}$ in diameter (Fig. 1). Preoperative differential diagnosis from Rathke's cyst and xanthogranuloma (Fig. 2) was necessary in four and 2 patients, respectively, and four of these were IS type tumors.

On histology, 22 cases $(68.8 \%)$ were adamantinomatous type, 7 cases were papillary type, and 3 cases were ciliated type craniopharyngioma.

\section{Surgical outcome}

Total resection of the tumor was achieved in 26 patients $(81.3 \%)$ and subtotal resection in 6 patients $(18.7 \%)$. In the latter 6 patients, firm adherence to the optic chiasm and surrounding arteries hindered total tumor resection. 5 were patients with a recurrent tumor (Table 2), 4 of whom had multiple surgeries. Total resection was achieved in all 7 patients with IS type, whereas total resection was achieved in only 19 patients $(76.0 \%)$ with SSE type (Table 1 ). 

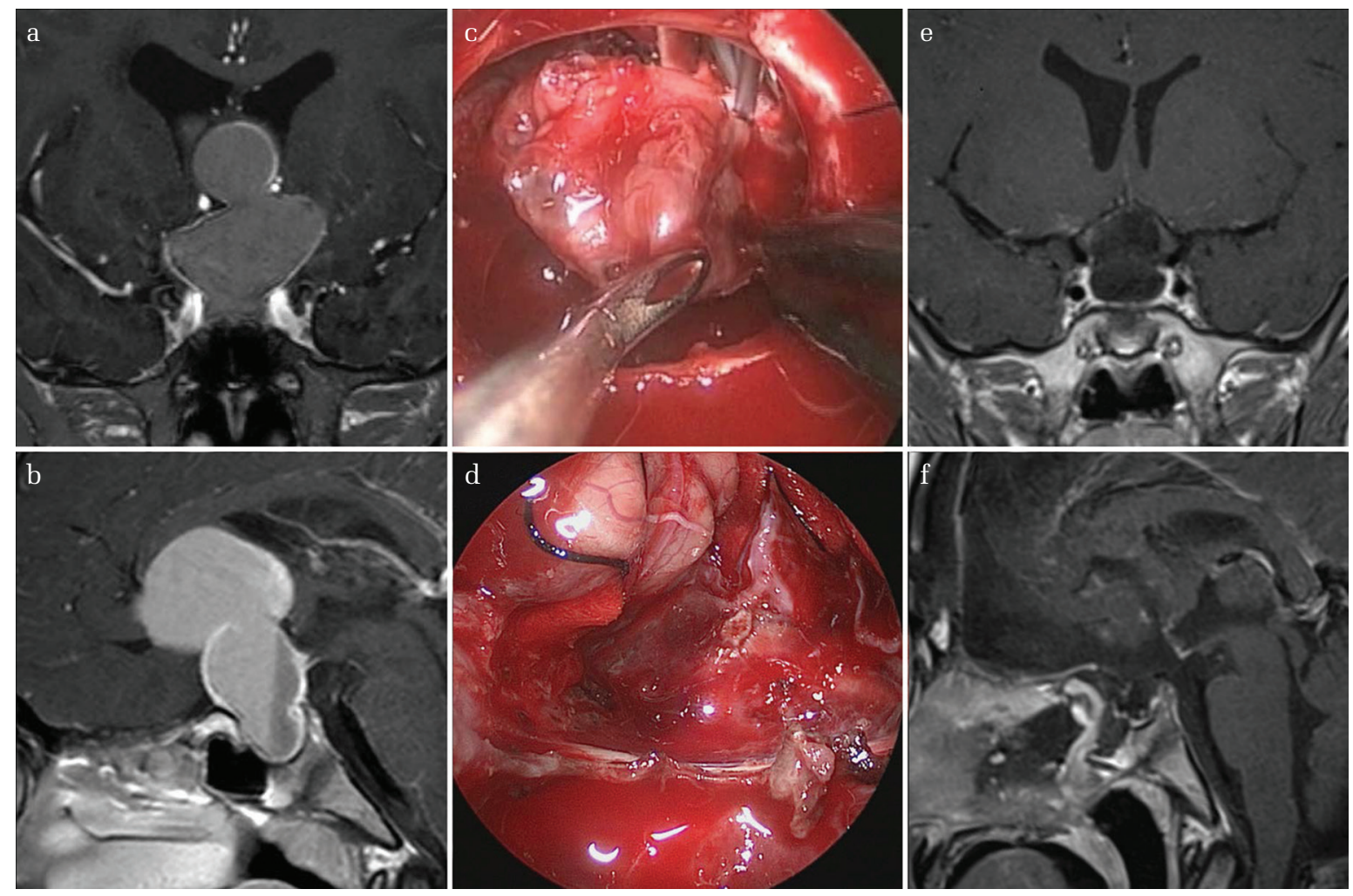

Fig. 1 Suprasellar extended type craniopharyngioma (adamantinomatous type) in an 8-year-old girl with preoperative hypopituitarism. The tumor was totally resected by combined transcranial-EES. The stalk was sacrificed and diabetes insipidus developed after surgery. Gadolinium-enhanced coronal (a and e) and sagittal (b and $f$ ) magnetic resonance images before ( $a$ and $b$ ) and after (e and f) surgery. Intraoperative view during (c) and after tumor removal (d).

Among the 27 patients with preoperative visual impairment, vision improved in 20 patients $(74.1 \%)$, remained unchanged in six patients $(22.2 \%)$ and deteriorated in one patient $(3.7 \%)$. In seven patients with no improvement or deterioration of visual function after surgery, 6 patients had a SSE type tumor and 6 patients were recurrent cases (Tables 1 and 2).

Apart from pituitary dysfunction and visual deterioration, no complications developed after surgery. 2 patients underwent adjuvant stereotactic cyberknife radiotherapy for the residual tumor. No patients experienced tumor recurrence during the mean follow-up period of 30.2 months.

\section{Endocrinological outcome}

Before surgery, 25 patients had hypopituitarism (defined as those who required replacement treatment) and 9 patients had diabetes insipidus. $15(71.4 \%)$ of the former and $5(23.8 \%)$ of the latter patients underwent primary surgery. An anatomically complete preservation of the pituitary stalk was achieved in $17(53.1 \%)$ of all patients and in $12(57.1 \%)$ of the 21 patients who underwent primary surgery (Table 2). The pituitary stalk was preserved in 3 patients $(42.9 \%)$ with IS type (Fig. 2) and in 14 patients $(56.0 \%)$ with SSE type (Fig. 3) (Table 1). In 4 patients with IS type, the stalk was sacrificed because direct invasion of the tumor was confirmed on intraoperative histological diagnosis. In the 7 patients without preoperative hypopituitarism, adenohypophysial function remained normal in 5, but new hypopituitarism developed after surgery in 2 patients, whose stalks were preserved. New diabetes insipidus, either partial or complete, developed after surgery in 17 patients (73.9\%), 9 of whom achieved stalk preservation. Finally, despite stalk preservation in 17 patients, adenohypophysial and neurohypophysial functions were completely preserved in only 5 and 6 patients, respectively.

\section{Discussion}

In the present study, subdiaphragmatic type tumors accounted for approximately $30 \%$ of all craniopharyngiomas. They showed a preponderance in young patients; $60 \%$ of patients were younger than 


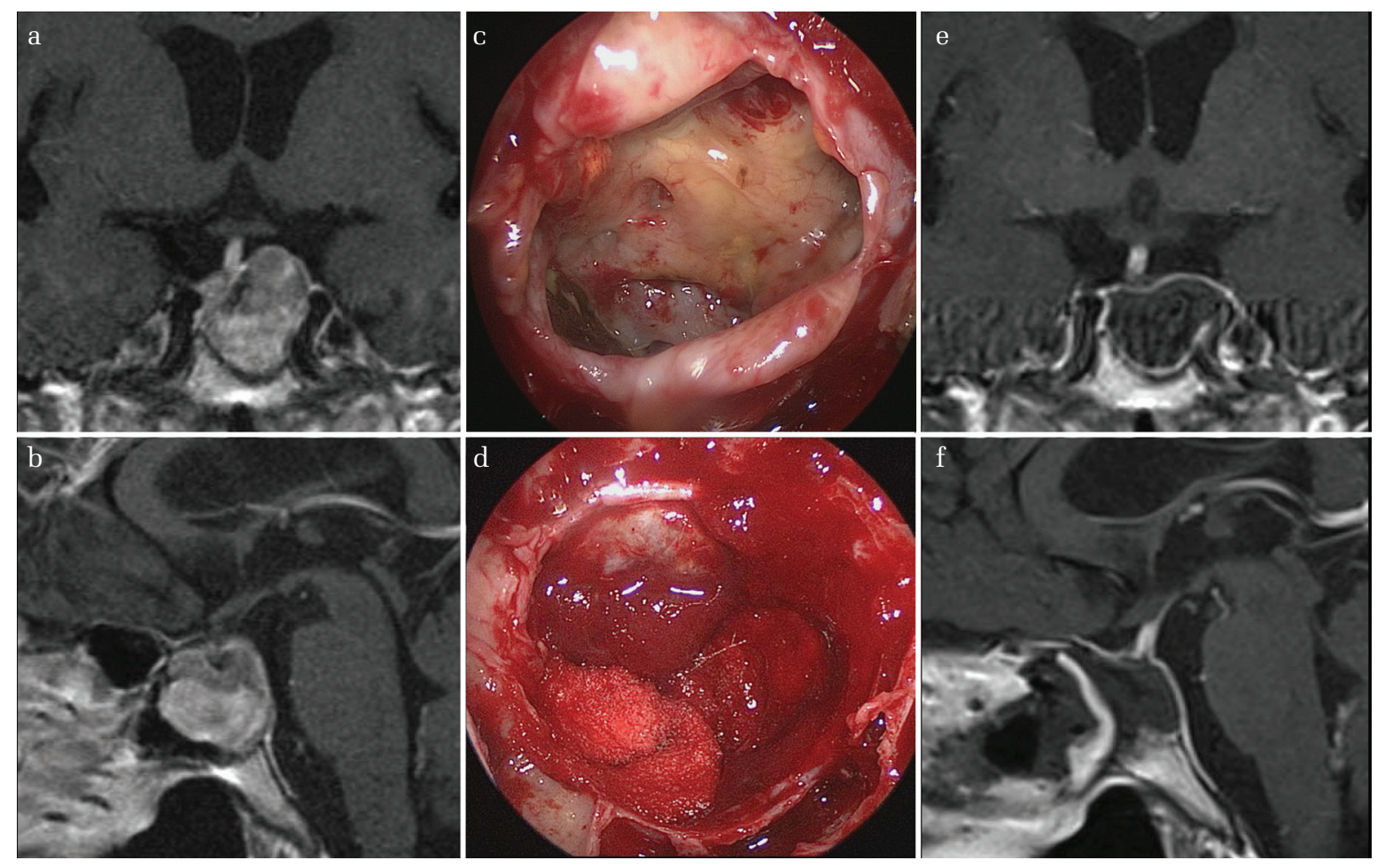

Fig. 2 Intrasellar type craniopharyngioma (papillary type) in a 69-year-old woman with preoperative hypopituitarism. The tumor was totally resected by conventional EES without complications. The stalk was preserved. Gadolinium-enhanced coronal (a and e) and sagittal ( $b$ and $f$ ) magnetic resonance images before (a and b) and after (e and f) surgery. Intraoperative view before (c) and after tumor removal (d).
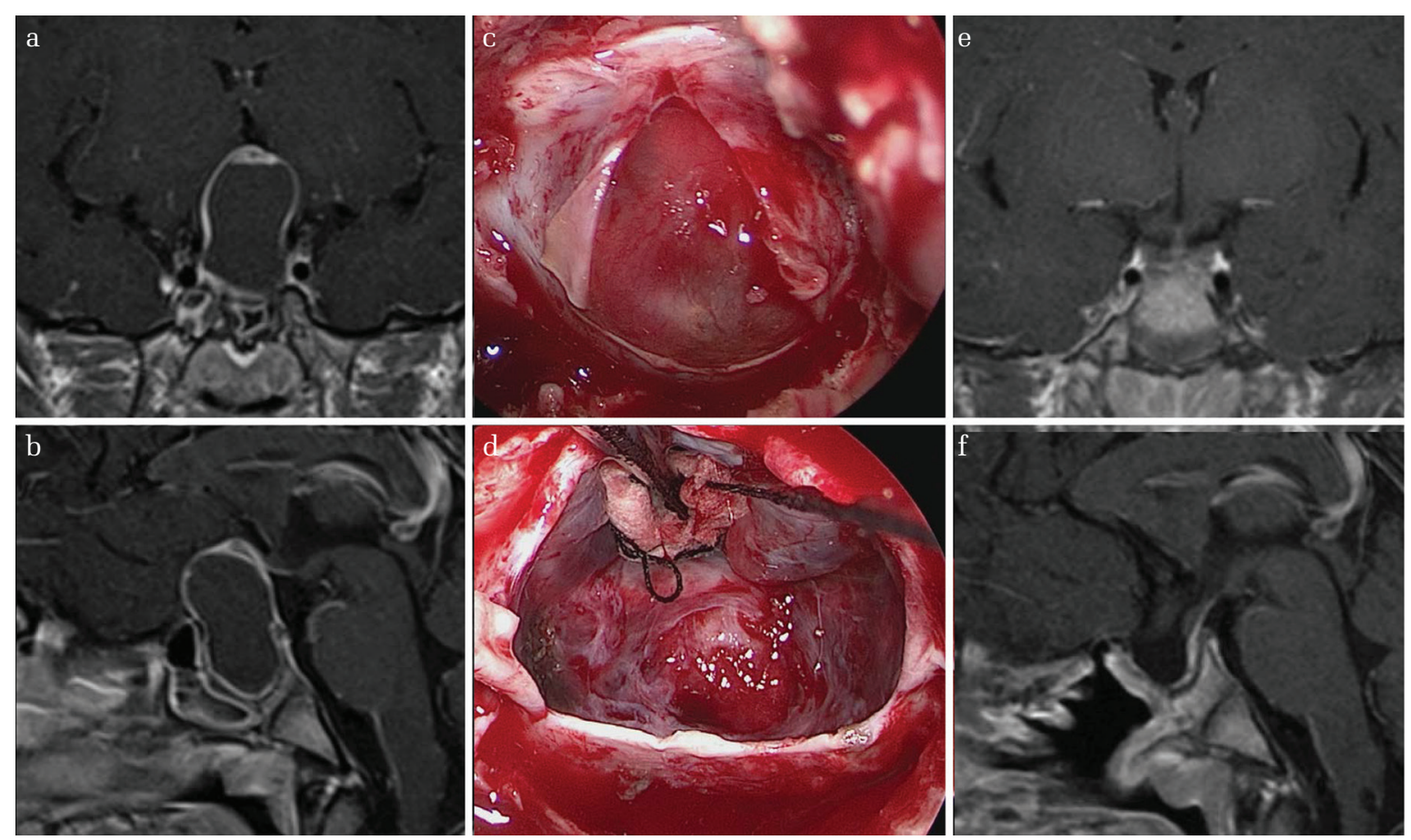

Fig. 3 Suprasellar extended type craniopharyngioma (adamantinomatous type) in an 11-year-old boy with preoperative hypopituitarism. The tumor was totally resected by conventional EES with preservation of the stalk but diabetes insipidus developed after surgery. Gadolinium-enhanced coronal (a and e) and sagittal (b and f) magnetic resonance images before ( $a$ and $b$ ) and after ( $e$ and f) surgery. Intraoperative view before (c) and after tumor removal (d). 
18-year-old. Most of the tumors were cystic, with more than half being almost entirely cystic. Thus, the tumors that were small and/or localized within the sella (i.e. an IS type) mimicked Rathke's cleft cyst. However, many of the tumors showed calcification on CT and nearly $70 \%$ of the tumors were adamantinomatous type on histology. In SSE type, suprasellar tumors were always extending ventrally to the stalk, and thus often showed growth to a prechiasmatic space with significant subfrontal/ frontal extensions.

Subdiaphragmatic type craniopharyngiomas are those originating within the sella. In general, their growth pattern is similar to those of pituitary adenomas. ${ }^{4,8}$ They usually accompany an enlarged sella. The suprasellar extended portion of the tumor is basically covered and demarcated by an elevated and stretched diaphragm (i.e. an extraarachnoidal tumor) unless it is destroyed by previous surgery. ${ }^{4-9)}$ TSS has been used as the most suitable surgical approach in most patients because these tumors can be treated similarly to pituitary adenomas. Previously, to avoid high-flow cerebrospinal fluid leakage, indication of TSS had been restricted to tumors located almost entirely within an enlarged sella (IS type) or those with a slight suprasellar extension. ${ }^{5-7)}$ In the literature, approximately $10-30 \%$ of craniopharyngiomas belonged to this indication. Following recent development of the extended approach and EES, most patients with subdiaphragmatic type became good candidates for EES. ${ }^{1-4)}$ When the suprasellar extended portion of the tumor is completely covered by an elevated and stretched diaphragm and remains as an extraarachnoidal tumor, the risk of intracranial vascular, nerve, and brain injuries are considerably lower than those of supradiaphragmatic type.

The diaphragm sellae can be extremely extended by enlargement of the tumor or can be destroyed by previous surgeries. In such cases, conventional TSS is insufficient for effective and safe tumor resection. In the present series, extended EES and combined transcranial-ESS were performed in 18 $(56.3 \%)$ and $3(9.4 \%)$ patients, respectively. The latter 3 tumors were extremely large $(44.5,53.4$, and $63.3 \mathrm{~mm}$ in maximum diameter) and showed significant frontal extension. There is a report that in subdiaphragmatic type, TSS could be reasonably attempted even with considerable suprasellar extensions because the superior surface of the tumor may be easily separated from the brain structures by pulling. ${ }^{8)}$ However, we believe that tumors extending anywhere beyond the safe range of surgical instruments should not be treated endonasally. ${ }^{1,2,9)}$ Either a transcranial approach or a combined approach is recommended for effective and safe resection of a few giant tumors with significant frontal extension. The 6 patients with subtotal tumor resection had a SSE type tumor, and 5 of these were recurrent cases. These cases showed tight adherence to the surrounding important tissues. 6 of 7 patients with no improvement or deterioration of visual function were recurrent cases as well.

In general, surgical outcomes of subdiaphragmatic type craniopharyngioma in terms of the extent of tumor resection was satisfactory in the previous surgical series. ${ }^{1-6)}$ Total or subtotal resection was achieved in most cases, particularly in IS type. These tumors originate within the sella, presumably from the distal end of the stalk or neurohypophysis. ${ }^{4,5,7,8)}$ Thus, anatomical preservation of the pituitary stalk can more frequently be achieved following radical tumor resection in this type than the supradiaphragmatic type. In the present series, total resection was achieved in more than $80 \%$ of cases and subtotal resection was achieved in the remaining cases. The pituitary stalk was anatomically preserved after surgery in more than half of the patients. However, pituitary dysfunctions were frequently observed both before and after surgery. Pituitary dysfunction, particularly hypopituitarism, was a common observation at initial presentation. New pituitary dysfunction, particularly diabetes insipidus, developed frequently after surgery irrespective of the tumor being IS or SSE type. Pituitary functions remained normal in approximately one third of patients with stalk preservation.

In patients with deterioration of pituitary function after surgery, the pituitary was probably damaged during dissection of the tumor from the distal end of the stalk or neurohypophysis. It has been reported that the tumor may firmly adhere to this site from which the tumor often originate, and thus complete tumor resection may carry considerable risk of diabetes insipidus. ${ }^{5,7)}$ Although the regrowth or recurrence of the tumor usually occurs within the sella (i.e. from this site), sharp excision from the stalk resulting in a subtotal removal may be required to avoid pituitary dysfunction in some patients with normal function before surgery. ${ }^{5,7)}$

\section{Conclusion}

Endoscopic endonasal surgery was best applied for subdiaphragmatic type craniopharyngiomas but some SSE type tumors required an extended or combined approaches. Surgical outcomes in terms of extent of tumor resection and complication were satisfactory, particularly in patients who underwent primary surgery. They are basically an extraarachnoidal 
tumor, and thus a stretched diaphragm usually demarcated the tumor from suprasellar structures. In some patients with recurrence, however, the tumor adhered to important suprasellar tissues. In contrast, the endocrinological outcome remained unsatisfactory despite stalk preservation in some patients. In patients without preoperative pituitary dysfunction, sharp excision of the area of firm adherence to the stalk and neurohypophysis should be considered.

\section{Acknowledgment}

Some pediatric patients in this study have been included in the previous study: S. Yamada, et al. Therapeutic outcomes of transsphenoidal surgery in pediatric patients with craniopharyngiomas: a single center study. J Neurosurg: Pediat (in press).

\section{Conflicts of Interest Disclosure}

There are no conflicts of interest, no commercial relationships, no support from pharmaceutical or other companies. The authors have no personal or institutional financial interest in drugs, materials, or devices described in the present paper.

\section{References}

1) Cavallo LM, Frank G, Cappabianca P, et al.: The endoscopic endonasal approach for the management of craniopharyngiomas: a series of 103 patients. J Neurosurg 121: 100-113, 2014

2) Cavallo LM, Solari D, Esposito F, Villa A, Minniti G, Cappabianca P: The role of the endoscopic endonasal route in the management of craniopharyngiomas. World Neurosurg 82: S32-S40, 2014
3) Koutourousiou M, Gardner PA, Fernandez-Miranda JC, Tyler-Kabara EC, Wang EW, Snyderman CH: Endoscopic endonasal surgery for craniopharyngiomas: surgical outcome in 64 patients. J Neurosurg 119: 1194-1207, 2013

4) Morisako H, Goto T, Goto H, Bohoun CA, Tamrakar S, Ohata K: Aggressive surgery based on an anatomical subclassification of craniopharyngiomas. Neurosurg Focus 41: E10, 2016

5) Abe T, Ludecke DK: Recent results of primary transnasal surgery for infradiaphragmatic craniopharyngioma. Neurosurg Focus 3: e4, 1997

6) Abe T, Lüdecke DK: Transnasal surgery for infradiaphragmatic craniopharyngiomas in pediatric patients. Neurosurgery 44: 957-964; discussion 964-966, 1999

7) Fahlbusch R, Honegger J, Paulus W, Huk W, Buchfelder M: Surgical treatment of craniopharyngiomas: experience with 168 patients. J Neurosurg 90: 237-250, 1999

8) Wang KC, Hong SH, Kim SK, Cho BK: Origin of craniopharyngiomas: implication on the growth pattern. Childs Nerv Syst 21: 628-634, 2005

9) Yamada S, Fukuhara N, Oyama K, et al.: Surgical outcome in 90 patients with craniopharyngioma: an evaluation of transsphenoidal surgery. World Neurosurg 74: 320-330, 2010

10) Horiguchi K, Nishioka H, Fukuhara N, YamaguchiOkada M, Yamada S: A new multilayer reconstruction using nasal septal flap combined with fascia graft dural suturing for high-flow cerebrospinal fluid leak after endoscopic endonasal surgery. Neurosurg Rev 39: 419-427, 2016

Address reprint requests to: Hiroshi Nishioka, MD, PhD, Department of Hypothalamic and Pituitary Surgery, Toranomon Hospital, 2-2-2 Toranomon, Minato-ku, Tokyo 105-8470, Japan. e-mail: nishioka@tokyo-med.ac.jp 